\title{
Patient Compton Scattered Radiation for Monitoring Prostate Radiotherapy with Gold Fiducial Markers: A Simulation Study
}

\author{
Ana Luísa Lopes, Hugo Simões, Carolina Travassos, and Paulo Crespo \\ LIP-Laboratório de Instrumentação e Física Experimental de Partículas, Department of Physics, University of Coimbra, \\ Coimbra, Portugal \\ Email: \{ana.lopes, hugo.simoes, carolina.travassos, crespo\}@ coimbra.lip.pt
}

\begin{abstract}
Orthogonal ray imaging is a new low-dose imaging technology under study aimed at assist externalbeam radiotherapy (EBRT) treatments. It consists in detecting radiation scattered in the patient and emitted perpendicularly to the incident beam. Such radiation can be collected by positioning a multi-sliced, collimator-based, 1D detector system parallel to the beam axis, hence collecting such orthogonal rays, or by using a pinhole-like detector system positioned above or below the patient. This system can potentially be useful for on-board imaging, or real-time EBRT monitoring. In terms of prostate cancer irradiation, the use of implanted fiducial markers allows a more precise verification of the gland position relative to the bony anatomy. The prostate target normally exhibits intra- and interfraction motion induced by the daily variation in rectal and bladder filling. In order to solve this issue, three gold fiducial markers are inserted into the prostate. In the present study, the imaging capability provided by utilizing $\mathrm{X}$-rays escaping the patient/fiducial markers orthogonally in respect to the incoming beam direction is studied by GEANT4 simulation. The results allow concluding that the signal provided by such $\mathrm{X}$-rays is highly coincident with the position of the gold fiducial markers, even in situations where these have been deviated by as little as $3.0 \mathrm{~mm}$.
\end{abstract}

Index Terms -image-guided radiotherapy, OrthoCT, Monte Carlo simulation, gold fiducial markers, prostate cancer

\section{INTRODUCTION}

OrthoCT (orthogonal computed tomography based on the collection of X-rays emitted orthogonally in respect to the incoming beam) is a novel imaging system under study here for potentially assisting external-beam radiotherapy (EBRT) treatments. First experiments have been carried out with single-pixel scanning along a heterogeneous muscle-like phantom [1] together with different Monte Carlo simulations [2], [3] performed at LIP in close collaboration with the University of Coimbra, the Portuguese Oncology Centers of Porto and Coimbra, and the Radiotherapy Department of Coimbra University Hospital Center. This proposed imaging technique consists in operating a dedicated X-ray detection system

Manuscript received March 4, 2017; revised July 4, 2017. specially adapted to collect selected patient-scattered radiation, aiming at: (1) imaging the tumor region with the patient already positioned for treatment (so-called onboard imaging), and/or (2) imaging in real-time the anatomic region under irradiation, including the tumor. The detection can be achieved by positioning one or more collimator-based, 1D detector systems parallel to the beam axis, hence collecting such orthogonal rays. The concept is presented in Fig. 1, which shows the OrthoCT system applied to monitor a lung irradiation. This technique provides computed tomography (CT)-like images without the need to rotate the X-ray source around the imaging patient, since it is based on the detection of the photons emitted at almost right angles with respect to the incoming photon flux. By applying a small, pencillike beam scanned in one or more known direction(s), the third point where the interaction occurred is given by the detector slice that is triggered, hence yielding a signal proportional to the photons escaping the patient (i.e. a signal correlated with the patient morphology). The OrthoCT system proposed may be parked inside the rotating gantry [3], which allows it to be used in complement to other image-guided radiotherapy (IGRT) techniques, seemingly a non-negligible advantage.

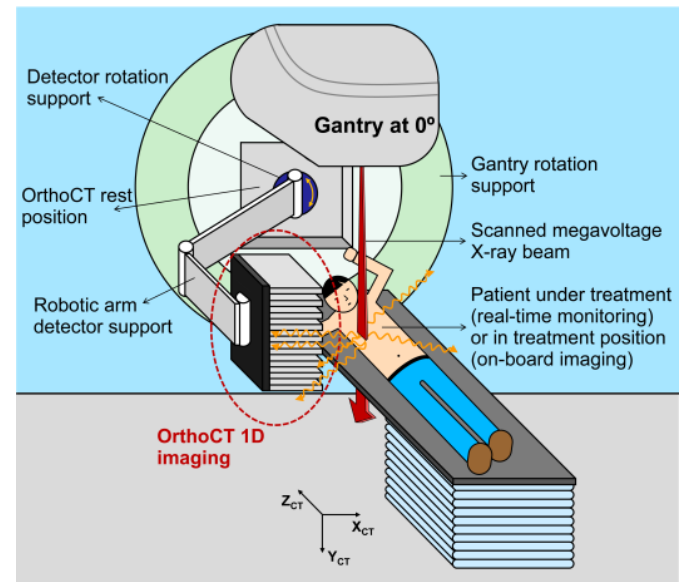

Figure 1. OrthoCT concept applied to a lung irradiation. The bidimensional position of the irradiation beam combined with the detector slice triggered by the scattered photons provides tridimensional information of the patient morphology just prior or during an EBRT treatment [3]. 
One of the main EBRT goals is to deliver the radiation dose as planned while minimizing the dose received by the surrounding healthy tissues and organs at risk. Thus, knowledge of the tumor location at the time of the treatment is crucial to optimize therapy and minimize possible side effects. This requirement is specially relevant to new radiotherapy techniques that involve dose escalation and hypofractionation, for example [4]. However, internal organ motion, possible morphological and anatomical changes and daily patient setup variations may prevent the desired dose from being delivered to the patient. In this context, simulation studies have been carried out to evaluate the feasibility of OrthoCT for monitoring possible variations that may occur in lung [3], [5] and head [6] irradiations. For both cases, the final results are very promising since the technique has shown to be fully capable of detecting tumor size variations, tumor mispositioning (Fig. 2), and also anatomical and/or morphological changes.

The capability of OrthoCT to monitor prostate irradiations has also been under assessment. Besides the techniques and protocols implemented, the prostate gland continues to be a challenge for EBRT due to the motion of the surrounding organs. For example, the dimensions of the bladder base can affect the displacement of the prostate in the anterior-posterior dimension, with the superior movement of the bladder resulting in anterior prostate displacement [7]. Furthermore, soft tissues like prostate have poor resolution in some of the current IGRT systems. One way to enhance the visibility of the lesion is by injecting a contrast agent. Thus, the benefit of iodinated-contrast agent incorporation into the OrthoCT images was previously evaluated [8]. The results show to be impossible to detect the tumor and the prostate in any of the studied scenarios: before and after contrast agent incorporation. Given these results, the impact of using socalled fiducial markers (FMs) on the accuracy and reproducibility of daily prostate target alignment based on orthogonal ray images is assessed here. According to the literature, one of the main methods used in IGRT to control the possible prostate position variations is the placement of FMs inside the gland. This method has been in clinical use since the early 90s [9], [10] and relies on the assumption that the markers accurately reflect the position of the prostate and that they do not significantly migrate within the gland [11].
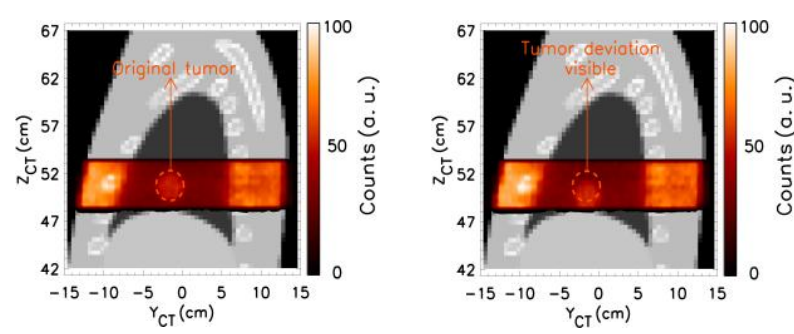

Figure 2. Simulated OrthoCT images obtained for a lung irradiation with the full collimated system. Left: tumor with the original size and positioning. Right: tumor deviated $9.36 \mathrm{~mm}$ in the craniocaudal direction. These images show a high visual agreement with the phantom morphology, allowing to detect the tumor dislocation. Adapted from [3].
The use of FMs allows to estimate the extent of variation in prostate position relative to external skin markings, from one fraction to another (interfraction motion), and during a single fraction (intrafraction motion) [12]. The most relevant desired properties substantiating the use of FMs are: (1) visibility in the several imaging systems involved in radiotherapeutic treatment planning and image guidance (e.g. CT, conebeam computed tomography, and electronic portal imaging devices), (2) low production of imaging artifacts, and (3) low disturbance of the therapeutic dose prescribed to the target volume [13].

In this work, the possible advantages in the display of gold FMs in OrthoCT images of the prostate are evaluated through GEANT4-based [14], [15] Monte Carlo simulations. For that a pelvis-like phantom was created and three gold objects were implemented inside the gland: one set had a spherical geometry [16] whereas the three other sets had a cylindrical one [17]. Finally, it must be stated that the OrthoCT technique being proposed here allows horizontal and/or slightly horizontal irradiations that do not trespass neither the bladder nor the colon/rectum due to its capability of obtaining 3D images without rotation of the X-rays source rotation (Fig. 3).

\section{MATERIALS AND METHODS}

The simulations were carried out with the pelvis-like phantom shown in Fig. 4 bottom. The phantom resembles in geometry and object density the anthropomorphic phantom NCAT [18] shown in Fig. 4 top. The low-energy electromagnetic physics package of GEANT4, version 9.3.p01 was utilized in the simulations. A 6-MeV endpoint energy spectrum (adapted from [19]) corresponding to a linac from Varian Medical Systems, Palo Alto, CA, EUA was implemented as input to the GEANT4-based simulation, since this photon energy is widely used in EBRT applications. The beam had a crosssection of $5 \mathrm{~mm} \times 5 \mathrm{~mm}$ performing a beam scan of $30 \mathrm{~mm}$ along the craniocaudal direction.

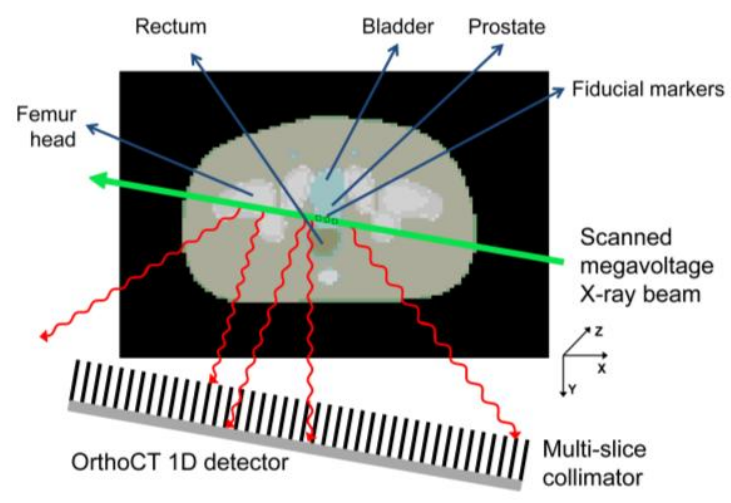

Figure 3. Scheme showing the positioning of the OrthoCT detector collecting some orthogonal rays in respect to the beam incoming direction. To notice is the fact that the scanned X-ray beam does not trespass rectum nor bladder. 

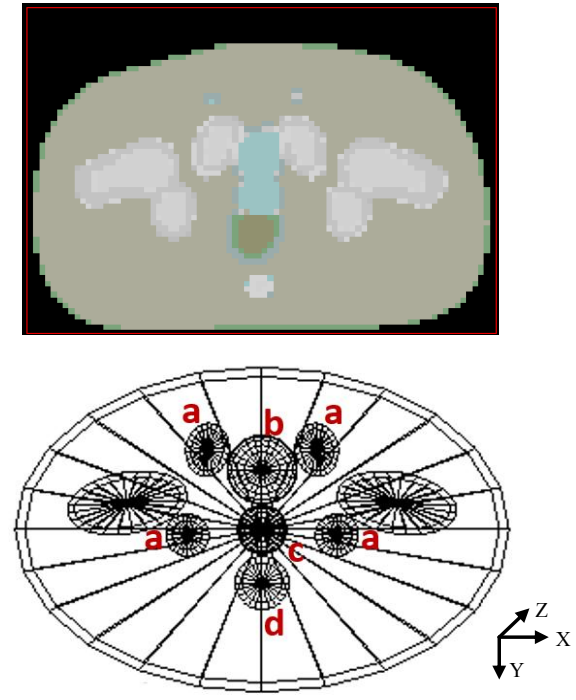

Figure 4. Axial views of the NCAT phantom (top) and the pelvis-like phantom (bottom) created for this study. a) Bone structures, b) bladder, c) prostate and d) rectum.

At this stage, all the OrthoCT images were created with mathematical collimation, i.e. only photons hitting the detector within an angle $\theta_{\mathrm{X}} \leq 1.5$ degrees and an energy $\mathrm{E}_{\gamma} \geq 200 \mathrm{keV}$ were considered to produce such images. To decrease some of the noise, a median filter with a span of 2 pixels was applied to all OrthoCT images. It should be pointed out that for all the four studied scenarios, the same setup and simulation parameters were applied into GEANT4 and a second simulation was carried out for each scenario with the FMs deviated 3.0 $\mathrm{mm}$ into the craniocaudal direction.

Due to the photon beam attenuation that occurs when it crosses the phantom, the orthogonal images present a higher intensity in the beam input region and a lower intensity in the beam output region. To homogenize the image intensity, a correction based on a line (with equation signal $_{\text {baseline }}=4 \times X-25$ ) was applied to each row of the final image.

\section{A. Three Golden Spheres with 2.0-mm Diameter}

First, three golden FMs with a spherical shape and 2.0$\mathrm{mm}$ diameter were implemented inside the prostate gland in different positions. According to the literature these dimensions are required for purposes of real-time radiotherapy monitoring [16]. This FMs dimension is slightly thicker than those normally used in clinical practice [17] which, in addition, present cylindrical geometries. For that, the studies shown hereafter comprise cylindrical FMs with dimensions typical of those utilized in a real clinical environment.

\section{B. Three Golden Cylinders with 1.6-mm Diameter and 3.0-mm Length}

The use of cylindrical gold FMs for prostate IGRT is standard practice in many centers. Normally, three small seeds are implanted inside the gland under transrectal ultrasound (TRUS) guidance. One of the typical sizes used for FMs is 1.6-mm diameter and 3.0-mm length [20], which are studied here for orthogonal ray imaging based on the scattering of X-rays emitted perpendicularly to the incoming therapeutic beam. In addition, in order to evaluate its visibility in OrthoCT images, the capability of this system to image photons escaping at 90 degrees in order to detect FMs deviations as small as $3.0 \mathrm{~mm}$ is also analyzed.

\section{Three Golden Cylinders with 1.2-mm Diameter and 3.0-mm Length}

In a third stage, a simulation study with FMs with a smaller diameter size was made. Here, the display of gold cylinders with 1.2-mm diameter and 3.0-mm length were assessed, since these are also one the most common dimensions used in clinical practice [17]. The remaining analyzes is similar to that of section II.B.

\section{Three Golden Cylinders with 1.0-mm Diameter and 5.0-mm Length}

Regarding the results obtained with the FMs described in section II.C, a final simulation study similar to that described in section II.B was made. The exception was the dimensions of the FMs which here presented a diameter of $1.0 \mathrm{~mm}$ and a length of $5.0 \mathrm{~mm}$. The aim of this study was to evaluate if the increased length of the FMs would influence the OrthoCT signal.

\section{RESULTS}

The OrthoCT images obtained with the pelvis-like phantom by means of mathematical collimation together with energy discrimination for all the studied scenarios are presented here, as well as their corresponding dose distributions (Fig. 5-8). As mentioned before, for each scenario two simulations were implemented: one with the FMs in their original position and a second one with a deviation of $3.0 \mathrm{~mm}$ in the craniocaudal direction. Indeed, it has been published [20] that interfractional deviations as large as $13.0 \mathrm{~mm}$ for prostate irradiations are possible.

\section{A. OrthoCT Images with Golden Spheres of 2.0-mm Diameter}

Fig. 5 top left shows the dose distribution obtained with an irradiation of $11 \mathrm{mGy}$ shot laterally to the patient, thus avoiding trespassing the bladder and the rectum/colon. In Fig. 5 bottom left the corresponding OrthoCT image shows clearly the position of three FMs. In addition, the images on the right column allow to infer that a prostate craniocaudal deviation of $3.0 \mathrm{~mm}$ has undoubtedly occurred as per the position of the FMs in the bottom right images.

\section{B. OrthoCT Images with Golden Cylinders of 1.6- $\mathrm{mm}$ Diameter and 3.0-mm Length}

Similarly to what was described in the previous paragraph, utilizing golden seeds with $1.6-\mathrm{mm}$ diameter and $3.0-\mathrm{mm}$ length allows the same conclusions to be drawn: a $3.0 \mathrm{~mm}$ deviation in the craniocaudal direction is clearly distinguishable (Fig. 6). 

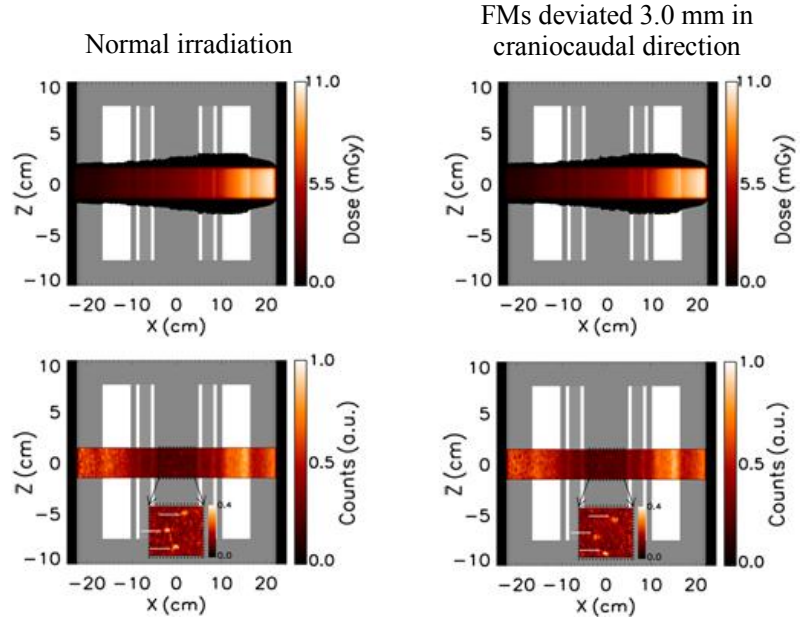

Figure 5. Coronal views of the simulated dose distributions (top) and OrthoCT images (bottom) for both studied scenarios with golden spheres with 2.0-mm diameter. The OrthoCT images clearly show the position of the three FMs in a normal irradiation (bottom left) and in a case of a deviation of $3.0-\mathrm{mm}$ in the craniocaudal direction (bottom right).

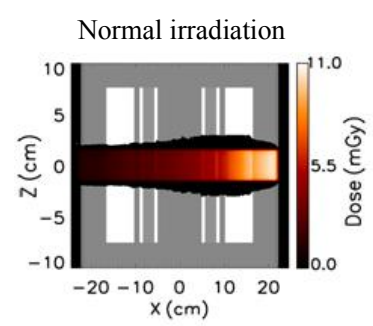

FMs deviated $3.0 \mathrm{~mm}$ in
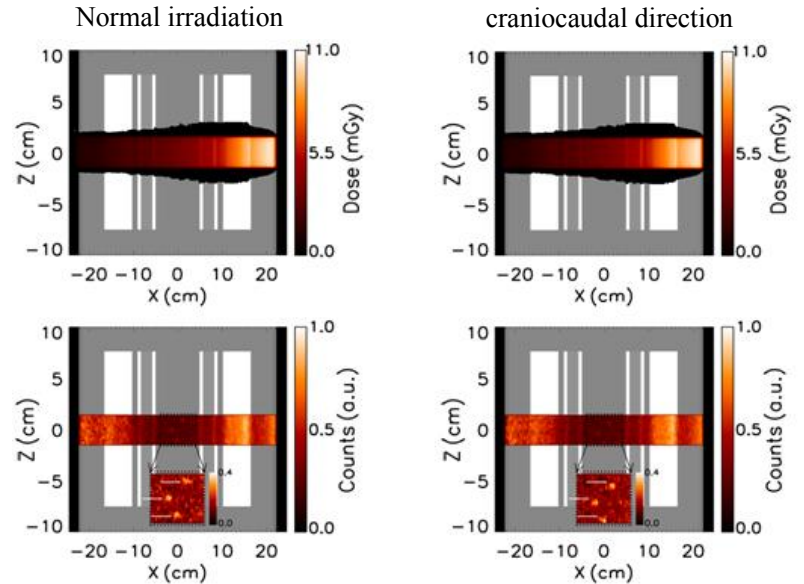

Figure 6. Coronal views of the simulated dose distributions (top) and corresponding OrthoCT images (bottom) for both studied scenarios with FMs of typical size and shape: cylinders of 1.6-mm diameter and 3.0mm length. It is possible to detect the position of the FMs in a normal irradiation (bottom left) and a 3.0-mm deviation in the craniocaudal direction is also clearly distinguishable (bottom right).

\section{OrthoCT Images with Golden Cylinders of 1.2-mm Diameter and 3.0-mm Length}

In what concerns the use of golden FMs with $1.2-\mathrm{mm}$ diameter and 3.0-mm length, Fig. 7 shows that with this smaller size of FMs the results are inconclusive for both studied scenarios. In fact, a brilliant peak appears at the location of the three FMs. However, many false positive peaks also appear in the image, which strongly disturbs its evaluation.

\section{OrthoCT Images with Three Golden Cylinders with 1.0-mm Diameter and 5.0-mm Length}

Finally, Fig. 8 shows that the use of even smaller FMs in diameter (although with larger length) does not allow to distinguish a normal irradiation scenario. Therefore these smallest FMs studied here are deemed as insufficient to be used with OrthoCT imaging.

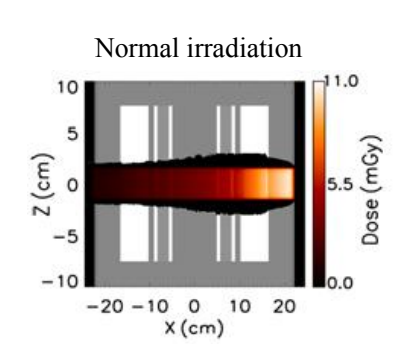

FMs deviated $3.0 \mathrm{~mm}$ in
craniocaudal direction
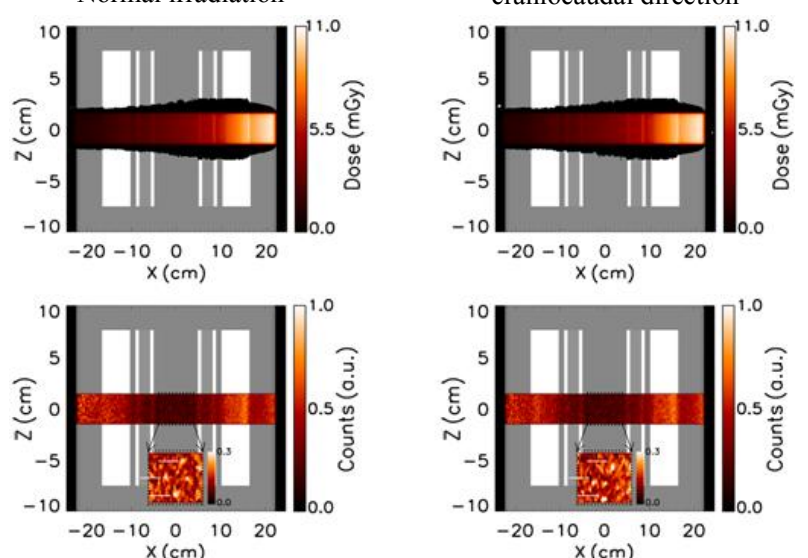

Figure 7. Coronal views of the simulated dose distributions (top) and corresponding OrthoCT images (bottom) for both studied scenarios with golden cylinders with 1.2-mm diameter and 3.0-mm length. In this case, the results are inconclusive since it is not possible to clearly distinguish the position of the FMs in a normal irradiation (bottom left) or in a situation where a deviation of $3.0 \mathrm{~mm}$ has occurred (bottom right) due to the presence of false positive peaks
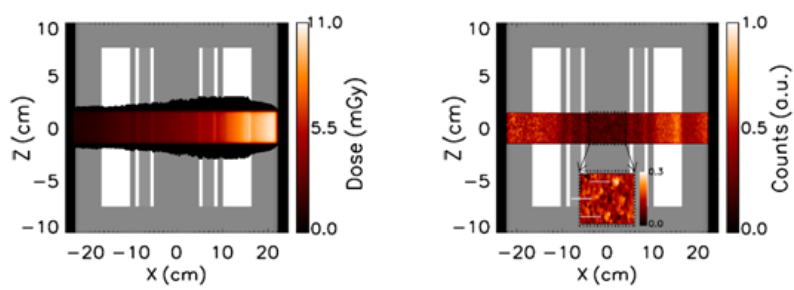

Figure 8. Coronal view of the simulated dose distribution (left) and corresponding OrthoCT image (right) for a scenario with golden cylinders with $1.0-\mathrm{mm}$ diameter and 5.0-mm length. In this case, the results are inconclusive since it is not possible to clearly distinguish the position of the FMs in this normal irradiation due to the presence of false positive peaks that fully disturb image evaluation. For this reason, the results obtained with deviated FMs are not shown.

\section{DISCUSSION AND CONCLUSIONS}

In the present work, the feasibility of utilizing Compton scattered radiation arising from megavoltage incoming beams onto FMs typically used in prostate EBRT was analyzed by means of simulation. The technique (so far in the simulation study stage) consists in detecting X-rays that have Compton scattered in the patient or FMs and that are detected at 90 degrees with respect to the incoming beam direction. At this stage, the signal of such rays perpendicular to the therapeutic beam was evaluated in order to conclude whether there is relevant information that could be collected by a full collimator system. The results were quite positive for (1) spherical, golden FMs with 2.0-mm diameter and (2) cylindrical, golden FMs with 1.6-mm diameter and 3.0$\mathrm{mm}$ length. For a diameter equal or smaller than $1.2 \mathrm{~mm}$, the results are inconclusive due to the presence of false positive peaks (low specificity) which highly disturbs image evaluation, at least for the doses utilized in this study.

In this study it is also shown that, in the imaging scenarios deemed useful (Fig. 5 and 6), no significant dose shadow effect seems to appear. 
Finally, all OrthoCT images deemed useful have shown no artifacts whatsoever, which is a remarkable advantage in respect to other imaging techniques utilized in concomitance with FMs [21].

Therefore it is possible to conclude that it is now worth to investigate the optimal multi-sliced collimator system that would allow to preserve the findings of this work. In parallel, a collimator based on a knife-edge slit could also provide a means to conserve the information gathered in this work.

Hence, in a forthcoming study the performance of both collimators in other to potentially obtain a device capable of monitoring prostate irradiations based on the implantation of FMs will be analyzed.

\section{ACKNOWLEDGMENT}

The authors acknowledge the informatics support from the staff of the high-performance computing cluster of the University of Coimbra. Research partly funded by FCT Fundação para a Ciência e a Tecnologia, Lisbon, Portugal, co-funded by the European Social Fund and by POPH Programa Potencial Humano (laboratory funding). Ana Luísa Lopes and Hugo Simões contributed equally to this work.

\section{REFERENCES}

[1] H. Simões, et al., "Dose-free monitoring of radiotherapy treatments with scattered photons: first experimental results at a 6 MV linac," IEEE Trans. Nucl. Sci., vol. 60, no. 4, pp. 3110-3118, 2013.

[2] M. C. Battaglia, H. Simões, V. Bellini, E. Cisbani, M. C. Lopes, and P. Crespo, "Orthogonal ray imaging with megavoltage beams: Simulated results with an anthropomorphic phantom," in Proc. Nuc. Sci. Symp. \& Med. Imag. Conf. (NSS/MIC), Anaheim, CA, 2012, pp. 3854-3859.

[3] H. Simões, et al., "Monitoring tumor lung irradiation with megavoltage patient-scattered radiation: A full system simulation study," IEEE Trans. on Radiation and Plasma Medical Sciences, 2017.

[4] T. R. Willoughby, et al., "Target localization and real-time tracking using the calypso $4 \mathrm{D}$ localization system in patients with localized prostate cancer," Int. J. Radiation Oncology Biol. Phys., vol. 65 , no. 2, pp. 528-534, Jan. 2006.

[5] H. Simões, M. Alves Barros, and P. Crespo, "OrthoCT for tumor lung irradiation: A simulation study," in Proc. Nuc. Sci. Symp. \& Med. Imag. Conf. (NSS/MIC), Strasbourg, France, 2016.

[6] H. Simões, A. L. Lopes, and P. Crespo, "OrthoCT for tumor head irradiation: A simulation study," in Proc. Nuc. Sci. Symp. \& Med. Imag. Conf. (NSS/MIC), Strasbourg, France: IEEE Press, 2016.

[7] J. Chen, R. J. Lee, D. Handrahan, and W. T. Sause, "Intensitymodulated radiotherapy using implanted fiducial markers with daily portal imaging: assessment of prostate organ motion," Int. J. Radiation Oncology Biol. Phys., vol. 68, no. 3, pp. 912-919, Feb. 2007.

[8] A. L. Lopes, et al., "Impact of tumor contrast in orthogonal ray imaging: a prostate irradiation study," in Proc. Nuc. Sci. Symp. \& Med. Imag. Conf. (NSS/MIC), Strasbourg, France, 2016.

[9] J. M. Crook, Y. Raymond, D. Salhani, H. Yang, and B. Esche, "Prostate motion during standard radiotherapy as assessed by fiducial markers," Radiother. and Oncol., vol. 37, pp. 35-42, July 1995.

[10] J. M. Balter, H. M. Sandler, K. Lam, R. L. Bree, A. S. Lichter, and R. K. T. Haken, "Measurement of prostate movement over the course of routine radiotherapy using implanted markers," Int. J. Radiation Oncology Biol. Phys., vol. 31, no. 1, pp. 113-118, 1995.
[11] J. Pouliot, et al., "(Non)-Migration of radiopaque markers used for on-line localization of the prostate with an electronic portal imaging device," Int. J. Radiation Oncology Biol. Phys., vol. 56, no. 3, pp. 862-866, 2003.

[12] D. Skarsgard, et al., "Planning target volume margins for prostate radiotherapy using daily electronic portal imaging and implanted fiducial markers," Radiat. Oncol., vol. 5, no. 52, 2010.

[13] D. Habermehl, K. Henkner, S. Ecker, O. Jäkel, J. Debus, and S. E. Combs, "Evaluation of different fiducial markers for imageguided radiotherapy and particle therapy," J. of Rad. Research, vol. 54, pp. i61-i68, 2013.

[14] S. Agostinelli, et al., "Geant4: a simulation toolkit," Nucl. Instrum. Meth. A, vol. 506, no. 3, pp. 250-303, 2003.

[15] J. Allison, et al., "Geant4 developments and applications," IEEE Trans. Nucl. Sci., vol. 53, no. 1, pp. 270-278, 2006.

[16] H. Shirato, et al., "Feasibility of insertion/implantation of 2.0-mmdiameter gold internal fiducial markers for precise setup and realtime tumor tracking in radiotherapy," Int. J. Radiation Oncology Biol. Phys., vol. 56, no. 1, pp. 240-247, 2003.

[17] T. Gawlik-Jakubczak and M. Matuszewski, "Fiducial markers in the treatment of prostate cancer: Technique and short term observation," Oncol. Clin. Pract., vol. 11, no. 6, pp. 295-299, 2015.

[18] W. P. Segars, "Development of a New Dynamic NURBS-based cardiac-torso (NCAT) Phantom," Ph.D. dissertation, The University of North Carolina, May 2001.

[19] F. Verhaegen and J. Seuntjens, "Monte Carlo modelling of external radiotherapy photons beams," Phys. Med. Biol., vol. 48, pp. R107-R164, 2003.

[20] G. Pang, D. J. Beachey, P. F. O’Brien, and J. A. Rowlands, "Imaging of 1.0-mm-diameter radiopaque markers with megavoltage X-rays: an improved online imaging system," Int. J. Radiation Oncology Biol. Phys., vol. 52, no. 2, pp. 532-537, 2002.

[21] M. F. Chan, G. N. Cohen, and J. O. Deasy, "Qualitative evaluation of fiducial markers for radiotherapy imaging," Technol. Cancer Res. Treat., vol. 14, no. 3, pp. 298-304, 2015.

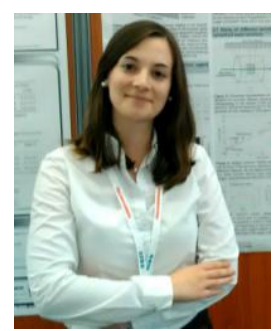

Ana Luísa Lopes received her BSc. in Radiotherapy in 2014 by the School of Allied Health Sciences of Porto, Portugal, and her MSc. in Biomedical Sciences in 2016, issued by the University of Beira Interior, Portugal. Since then she has been working as a researcher at LIP - Laboratório de Instrumentação e Física Experimental de Partículas, Coimbra, Portugal developing Monte Carlo software in order to evaluate the impact of using contrast agents and/or gold fiducial markers in orthogonal ray imaging. During the last year, she has co-authored two publications and one oral communication in International Conferences of IEEE. Her main areas of interest include radiotherapy, medical imaging and nuclear medicine regarding the development of new detectors and/or techniques to be applied in these areas of expertise.

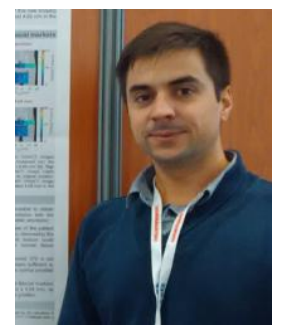

Hugo Simões received his MSc. degree in Biomedical Engineering from University of Coimbra, Portugal, in 2008. He is currently pursuing a Ph.D degree in Biomedical Engineering at University of Coimbra, Portugal. $\mathrm{He}$ has worked at Laboratório de Instrumentação e Física Experimental de Partículas, Coimbra, Portugal, since 2011, as a researcher in the Orthogonal Imaging concept, including real-time radiation therapy monitoring, and morphological imaging of targets. He has published some peer reviewed articles in IEEE Transactions on Nuclear Science and Journal of Instrumentation and several conference records in IEEE Medical Imaging Conference, among others. His research interests are in medical imaging, image processing, radiation therapy, and GEANT4 Monte-Carlo simulation. 


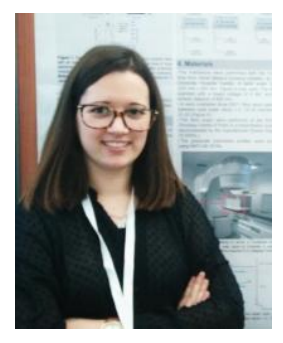

Carolina Travassos received her MSc. degree in Biomedical Engineering from the University of Coimbra, Portugal, in 2016.

She has been working at LIP - Laboratório de Instrumentação e Física Experimental de Partículas, Coimbra, Portugal as a researcher in the Orthogonal Ray Imaging project. She has published a conference record in IEEE Nuclear Science Symposium and Medical Imaging Conference 2016 and a chapter of the book Applied Physical Chemistry with Multidisciplinary Approaches, 2017. Her research interests are in medical imaging, image processing, radiation therapy, nuclear medicine and cancer disease.

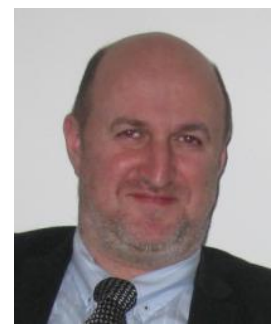

Paulo Crespo graduated in physics engineering by the University of Coimbra, Portugal, in 1997, and obtained his $\mathrm{PhD}$ in physics by the Technische Universität Darmstdat, Germany, in 2005, after some intermediate post graduate research at LIP Laboratório de Instrumentação e Física Experimental de Partículas - within the liquid-xenon PET project and also within the AMS collaboration (detector simulation and search for anti-matter).
$\mathrm{He}$ is a Professor at the Department of Physics of the University of Coimbra and Principal Investigator at LIP. He has co-authored 2 granted patent applications, 77 publications of which 38 in peerreviewed journals and 39 in international conference records with review. He has also co-authored 4 chapters in the books Nuclear Medicine Physics (New York, CRC Press \& Taylor \& Francis Group, 2010). He supervised two complete $\mathrm{PhD}$ works (other two ongoing) and supervised 10 complete MSc. works. Current interests include imaging in radiotherapy - particle or X-ray based, nuclear medicine with resistive plate chambers, and new methods for transcranial magnetic stimulation.

Prof. Crespo is a senior member of the IEEE society (NPSS and EMBS), the Deutsche Physikalische Gesellschaft, the Portuguese Physics Society and the European Physical Society. Prof. Crespo was awarded a highlight of 2006 by the IOP, a distinction to the top ten articles of largest impact according to the referees. Finally, Prof. Crespo is a member of the scientific committee of ICNAS (Institute of Nuclear Science Applied to Health) from the University of Coimbra. 